\title{
Successful Thrombolysis and Spasmolysis of Acute Leg Ischemia after Accidental Intra-arterial Injection of Dissolved Flunitrazepam Tablets
}

\author{
B. Radeleff - U. Stampfl - C.-M. Sommer • \\ N. Bellemann · A. Hyhlik-Duerr • M.-A. Weber • \\ D. Boeckler $\cdot$ H.-U. Kauczor
}

\begin{abstract}
A 37-year-old man with known intravenous drug abuse presented in the surgical ambulatory care unit with acute leg ischemia after accidental intra-arterial injection of dissolved flunitrazepam tablets into the right femoral artery. A combination of anticoagulation, vasodilatation, and local selective and superselective thrombolysis with urokinase was performed to salvage the leg. As a result of the severe ischemia-induced pain, the patient had to be monitored over the complete therapy period on the intensive care unit with permanent administration of intravenous fluid and analgetics. We describe the presenting symptoms and the interventional technique, and we discuss the recent literature regarding the management of accidental intra-arterial injection of dissolved flunitrazepam tablets.
\end{abstract}

Keywords Accidental intra-arterial injection Flunitrazepam · Leg ischemia - Spasmolysis .

Thrombolysis

\section{Case Report}

A young man (37 years old) with known intravenous drug abuse presented in the surgical ambulatory care unit with

\footnotetext{
B. Radeleff $(\bowtie)$ - U. Stampfl · C.-M. Sommer N. Bellemann - M.-A. Weber - H.-U. Kauczor

Diagnostic and Interventional Radiology, University of Heidelberg, Im Neuenheimer Feld 110, 69120 Heidelberg, Germany

e-mail: Boris_radeleff@med.uni-heidelberg.de
}

\section{A. Hyhlik-Duerr · D. Boeckler}

Department of Vascular and Endovascular Surgery, University of Heidelberg, Im Neuenheimer Feld 110, 69120 Heidelberg, Germany acute leg ischemia after accidental intra-arterial (right femoral artery) injection of dissolved flunitrazepam tablets (Rohypnol, Hoffmann-La Roche, Grenzach-Wyhlen, Germany) into the right femoral artery. The right leg was cold and painful with mottling of the skin in the lower leg. Motor function was impaired, but sensor function was not affected. Peripheral arterial pulses were present at the right-sided groin, popliteal fossa, and lateral malleolus.

The flunitrazepam tablets were crushed and dissolved in tap water (two tablets corresponding to $2 \mathrm{mg}$ of flunitrazepam). The injected powder may lead to distal thrombosis and spasms in the arteries and arterioles, in most cases without any surgical remedy. Therefore, we discussed the situation with vascular surgeons and decided to perform thrombolysis and spasmolysis to prevent subsequent tissue necrosis.

We consulted with the national center for coordinating emergency rescue in case of intoxication; the center recommended the intra-arterial application of lidocaine, prostaglandin, and urokinase in combination. This recommendation was based on their experiences after accidental intra-arterial injection of flunitrazepam into the brachial, radial, or ulnar arteries, which is much more common than in the lower extremities; however, it was comparable to the experiences with the lower extremities found in the recent literature [1-4].

\section{Technique}

The left femoral artery was punctured, and a short 4F sheath (Terumo Europe, Belgium) was placed. A 4F pigtail catheter (Cordis, Johnson \& Johnson, USA) was positioned in the abdominal aorta above the aortic bifurcation (Fig. 1). In the overview angiography, we depicted regular common, 
internal, and external iliac arteries on both sides without irregularities. Subsequently we performed a crossover placement of a 4F cobra-shaped catheter (Terumo Europe) into the right common iliac artery with a stiff hydrophilic 0.035 -inch guide wire (Terumo Europe). Over a heavy-duty wire (Amplatz Superstiff, Boston Scientific, Watertown, MA) we pushed a 6F Destination sheath (Terumo Europe) crossover into the right femoral artery. After intra-arterial application of $5000 \mathrm{IU}$ heparin, we catheterized the right superficial artery with the stiff hydrophilic 0.035 -inch Terumo guide wire with a 4F hydrophilic multipurpose catheter (Glidecath; Terumo Europe). Then we superselectively intubated the tibiofibular trunk and the anterior tibial artery with a $2.7 \mathrm{~F}$ Progreat microcatheter (Terumo Europe). After placement of the microcatheter in the distal tibial artery, angiography with simultaneous injection via the sheath and the microcatheter showed a slow, delayed runoff in the distal superficial artery and the right lower leg. The plantar artery was patent, but the dorsalis pedis artery was occluded. Therefore, we performed a superselective thrombolysis beginning with the intra-arterial injection of $20 \mu \mathrm{g}$ prostaglandin E1 (Prostavasin, Schwarz Pharma, Monheim, Germany) dissolved in $50 \mathrm{ml} 0.9 \% \mathrm{NaCl}, 100$ mg lidocaine (Xylocaine, AstraZeneca, Wilmington, DE) and $500 \mu \mathrm{g}$ glyceryl trinitrate (Nitrolingual, Pohl-Boskamp, Germany). Afterward, we injected a bolus of 500,000 IU urokinase (Urokinase Medac, Medac, Hamburg, Germany) and $20 \mathrm{mg}$ recombinant tissue plasminogen (r-tPA; Actilyse, Boehringer Ingelheim, Ingelheim, Germany). During this first intervention, we injected a total $1.4 \mathrm{mg}$ glyceryl trinitrate. Finally we noticed an improvement concerning the runoff, especially in the fibular and anterior tibial artery.

Thereafter, we referred the patient to the intensive care unit for surveillance. The thrombolysis was continued with heparinization (with a target partial thromboplastin time [PTT] of 50-60 s) and application of prostaglandin $20 \mu \mathrm{g}$ over $6 \mathrm{~h}$ via the $6 \mathrm{~F}$ sheath (positioned in the right femoral artery), with 60,000 IU urokinase per hour via the $4 \mathrm{~F}$ catheter (positioned above the anterior tibial artery) and injection of 60,000 IU urokinase per hour via the microcatheter positioned in the anterior tibial artery.

The control angiography after $8 \mathrm{~h}$ showed a good perfusion of the lower leg, with excellent runoff into the anterior tibial artery with a patent plantar artery; however, the dorsalis pedis artery was still occluded. The anticoagulation, thrombolysis, and spasmolysis were continued overnight as described above.

On the second day, angiography via the $6 \mathrm{~F}$ sheath showed a good runoff from the femoral artery to the lower leg (Fig. 2). The control angiography via the selective catheter showed much better perfusion in the lower leg, with good runoff in the anterior tibial artery with a patent plantar artery. The dorsalis pedis artery was still occluded.
The tip of the microcatheter was dislocated (due to nightly movements of the agitated patient) into the popliteal artery. Therefore, the microcatheter was removed, and the $4 \mathrm{~F}$ multipurpose catheter was positioned in the distal popliteal artery. The anticoagulation, thrombolysis, and spasmolysis was continued overnight with heparinization (target PTT 50-60 s) and application of prostaglandin E1 (20 $\mu$ g over $6 \mathrm{~h}$ ) via the $6 \mathrm{~F}$ sheath (positioned in the right femoral artery) and with 60,000 IU urokinase per hour over the $4 \mathrm{~F}$ catheter (positioned in the tibiofibular trunk).

On the third day, angiography showed patent vessels in the upper and lower leg, but the runoff in the lower leg and foot remained very slow (Fig. 3). The dorsalis pedis artery was still occluded, and the plantar artery showed a new filiform spasm. The anticoagulation, thrombolysis, and spasmolysis were continued overnight in the same manner as before.

After 3 days of therapy, the occlusions and spasms of the anterior tibial, dorsalis pedis artery, and plantar artery completely resolved, with patent vessels down to the foot (Fig. 4). After an interdisciplinary discussion with the inhouse vascular surgeons, we finished the intervention by removing the $6 \mathrm{~F}$ sheath in the left femoral artery with a vascular closure device (AngioSeal Evolution, St. Jude Medical, Little Canada, MN). We did not notice any of the typical complications such as bleeding or hematoma.

Because of the severe ischemia-induced pain, high doses of intravenous analgetics via central venous catheter access were necessary to enable the interventions. Therefore, the patient was supervised over the entire treatment period on the intensive care unit with monitoring of laboratory data, heart rate, and blood pressure and with permanent administration of intravenous fluid and analgetics.

Rhabdomyolysis is a common complication after accidental intra-arterial injection of dissolved flunitrazepam [1]. We did not noticed any elevation of serum creatinine $(<0.8 \mathrm{md} / \mathrm{dl})$. Renal function, electrolytes, and blood $\mathrm{pH}$ were frequently monitored and were not found to be affected at any time. Creatine kinase as a marker of the muscle tissue damage measured $38 \mathrm{U} / \mathrm{L}$ (normal range $<190 \mathrm{U} / \mathrm{L}) 2 \mathrm{~h}$ after the injection, with a maximum elevation to $4154 \mathrm{U} / \mathrm{L}$ after $24 \mathrm{~h}$. After the extraction of the sheath, the patient received low-molecular-weight heparin in prophylactic doses. By the time of discharge (day 8 after the start of therapy), clinical status and function of the right leg were completely restored without any sensory or residual motor loss. A compartment syndrome or tissue necrosis was not clinically present at any time.

\section{Discussion}

The accidental intra-arterial injection of dissolved tablets including benzodiazepines is a severe complication 

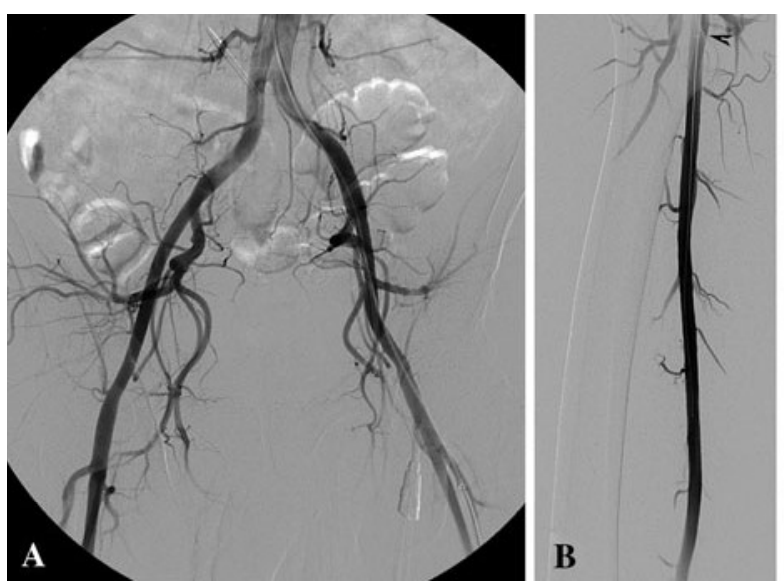

Fig. 1 A Overview angiography via a $4 \mathrm{~F}$ pigtail catheter in the abdominal aorta above the aortic bifurcation showing regular common, internal, and external iliac arteries on both sides without irregularities. B After a crossover maneuver, a 6F Destination sheath (black arrowhead) was placed into the right superficial artery. C Slow, delayed runoff in the distal superficial artery with $\mathbf{D}$ missing uptake in the right lower leg. The dorsalis pedis artery was occluded.
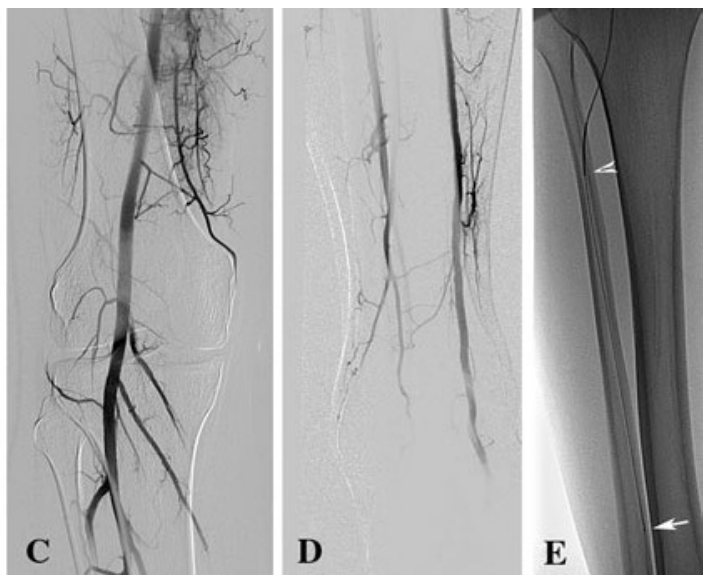

E Placement of a 2.7F Progreat microcatheter (white arrow) in the distal tibial artery via a $4 \mathrm{~F}$ hydrophilic multipurpose Glidecath catheter (white arrowhead). From this position, we performed a superselective spasmolysis and thrombolysis with intra-arterial injection of $20 \mu \mathrm{g}$ prostaglandin E1, $100 \mathrm{mg}$ lidocaine, and $500 \mu \mathrm{g}$ glyceryl trinitrate. Afterward we injected 500,000 IU urokinase and $20 \mathrm{mg} \mathrm{r-tPA}$ as a bolus
Fig. 2 Angiography at the second day of treatment showed good runoff from the femoral artery into the tibiofibular trunk. The control angiography via the selective catheter showed an improved perfusion in the lower leg with good runoff in anterior tibial artery, but the dorsalis pedis artery was still occluded. Anticoagulation, thrombolysis, and spasmolysis were continued
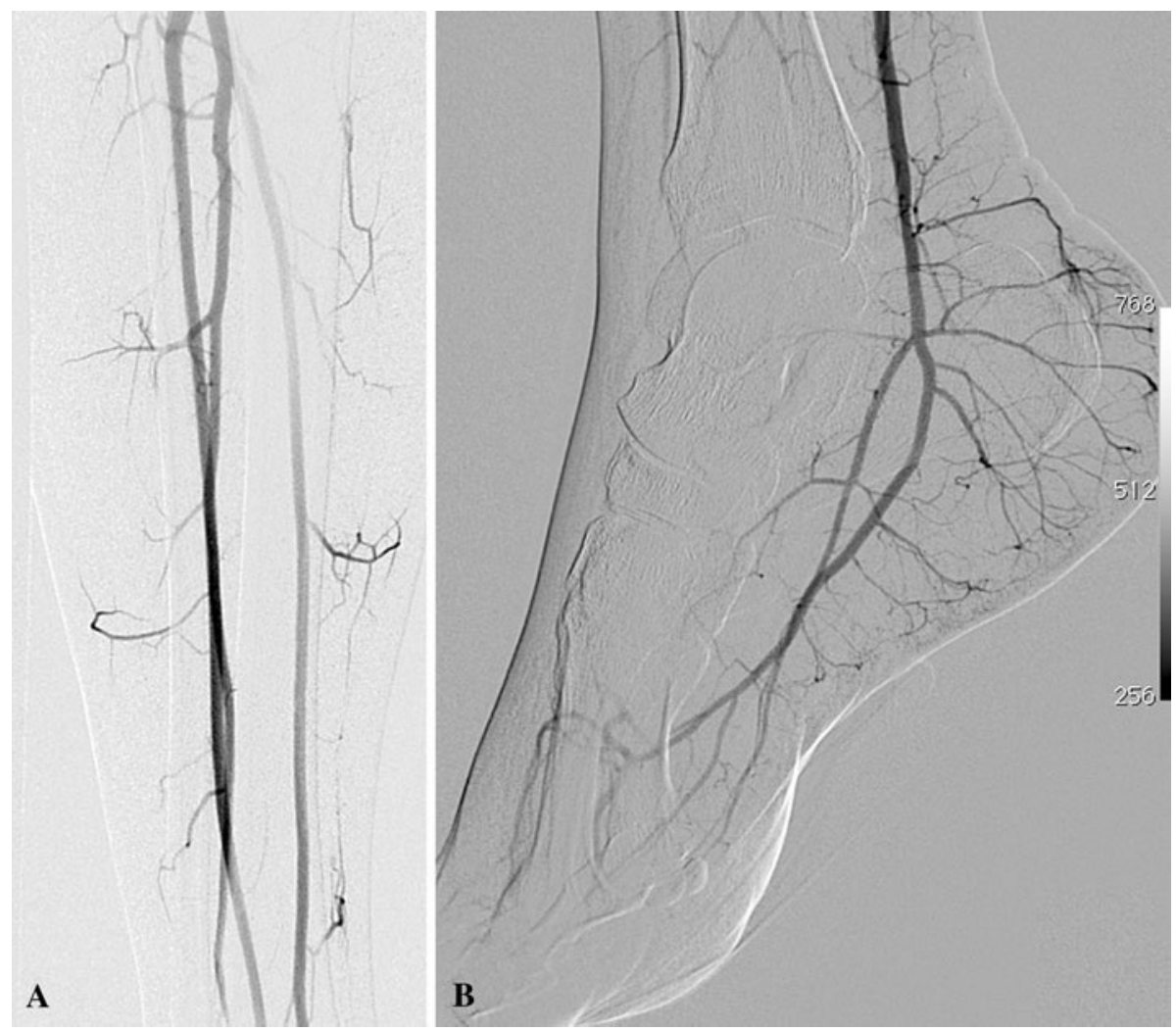

occurring in long time intravenous drug addicts. The attempted intravenous injection of benzodiazepines is performed to palliate the physical withdrawal symptoms. For this purpose, the use of diazepam, flunitrazepam, midazolam, or temazepam has been described [1,3-6]. The most common injection sites of accidental arterial administration are the arteries of the upper extremities as a result of the difficult venous access in the arm bend after long time intravenous drug abuse [1, 6-13].

The tablets dissolved in tap water contain multiple small particles of incompletely solubilized substances or even insoluble drug crystals, which can occlude precapillary 


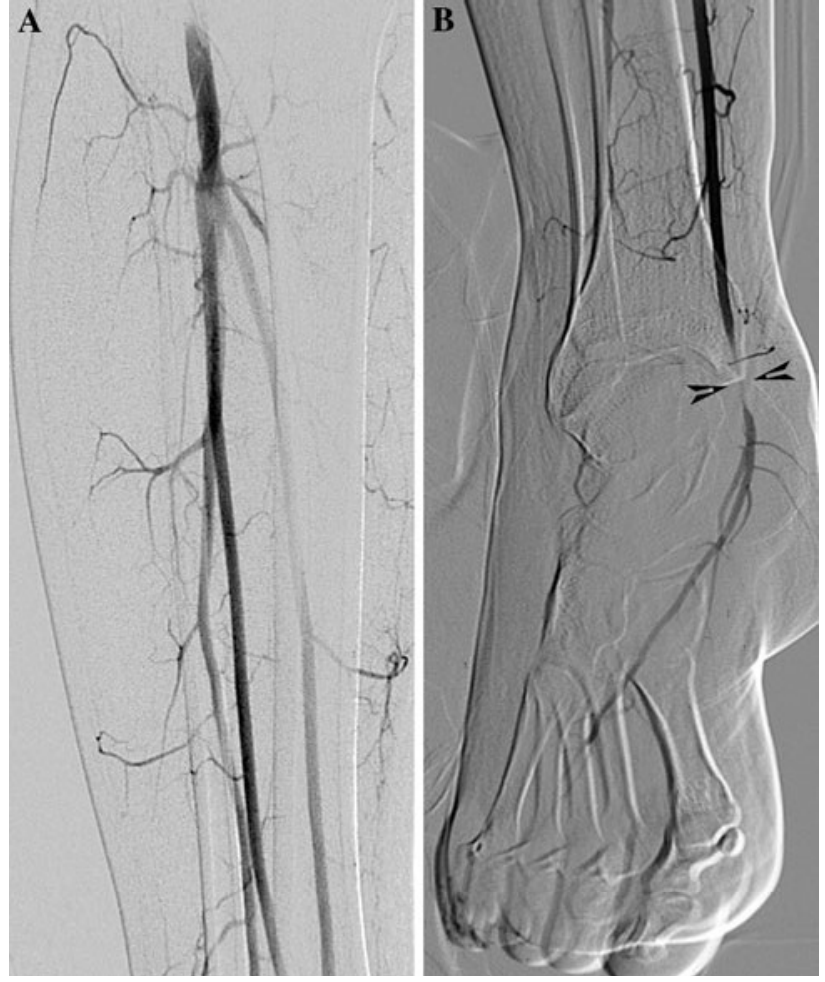

Fig. 3 Control angiography after 2 days showed patent vessels in the upper and lower leg, but the runoff in the lower leg and foot remained slow. The dorsalis pedis artery was still occluded, and the plantar artery showed a new filiform spasm. Therefore, anticoagulation, thrombolysis, and spasmolysis were continued

arterioles [13]. Beside the active component of the drug (leading to toxic endothelial damage and subsequent vascular thrombosis), many drugs contain microcrystalline cellulose [10]. All these debris particles together cause microvascular embolizations and massive spasms, followed by acute ischemia in the extremity, leading to severe pain almost immediately after the injection despite persistently palpable pulses $[1,2,13]$. The skin of the affected extremity often looks mottled (pallor; purplish and cyanotic areas); later, edema occurs, and tissue necrosis can develop $[1,2,13]$. Rhabdomyolysis is a common secondary complication, in some cases requiring temporary hemodialysis to treat renal failure if large muscles are involved [13].

The intra-arterial accidental injection of benzodiazepines regularly leads to distal thrombosis and spasms in the arteries and arterioles with little surgical options in most cases. The therapeutic strategy is not yet defined and depends on the symptoms and clinical findings. Intraarterial therapy may be indicated in early stages with ischemia but without prominent edema or even beginning rhabdomyolysis and tissue necrosis. As a result of the combination of microembolization and severe spasms, only combined anticoagulation, local thrombolysis, and vasodilatation might be successful to save the extremity $[1,9$, 14].

The application of heparin for anticoagulation (PTT $60-80 \mathrm{~s}$ ) is required to maintain patent collaterals and inhibit further expansion of possible thrombus [1, 6]. Most groups perform intravenous heparinization, but we favor the intra-arterial application of heparin via the sheath. Therefore, we used a perfusor system (25,000 IU heparin in $50 \mathrm{ml}$ running at $2.0 \mathrm{ml} / \mathrm{h}$ ) with a target PTT of $50-60 \mathrm{~s}$.

Local thrombolysis has been described in the literature either selectively, via the angiography catheter, or
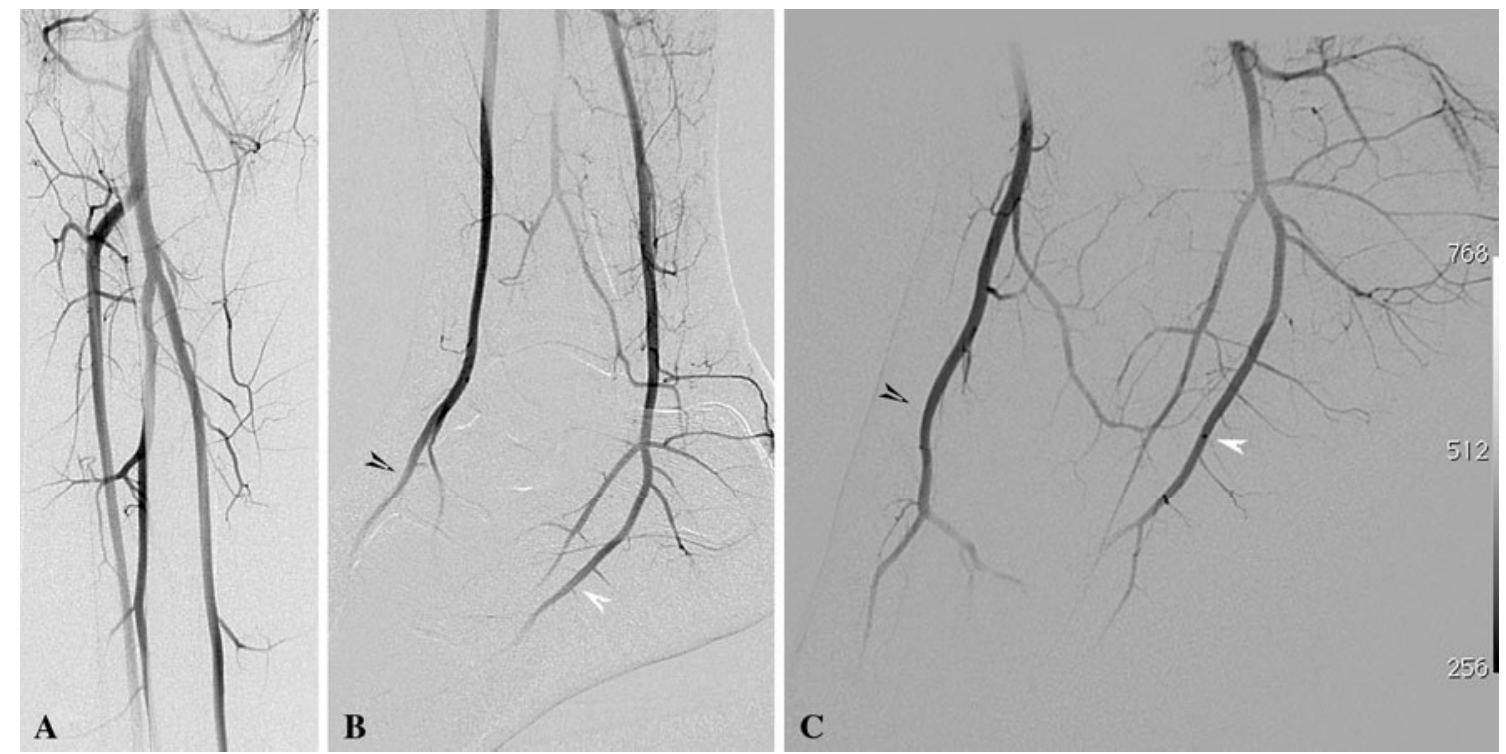

Fig. 4 After 3 days of therapy, the occlusions and spasms of the anterior tibial, dorsalis pedis artery, and plantar artery had completely resolved with patent vessels in the foot 
superselectively, via the microcatheter into the affected artery [5]. For thrombolysis, different medications have been used such as r-tPA, urokinase, and streptokinase, without one drug being better than the others [1, 8-11, 14].

For vasodilatation and spasmolysis, various medications have been used, such as calcium antagonists, nitrates, papaverine, and prostaglandins $[15,16]$. Especially the prostaglandins have different positive effects in this therapy because they are can repair endothelial dysfunction, stimulate the fibrinolytic activity, and provide a local antiinflammatory effect $[5,10]$. In addition, prostaglandins improve the lytic effect of urokinase and r-tPA [14]. Tait et al. [17] recommended a systemic therapy with prostacyclin $(2 \mathrm{ng} / \mathrm{kg} / \mathrm{min}$ for 48-96 h) with a dramatic improvement of distal limb perfusion within $12 \mathrm{~h}$ in their study of eight patients.

In terms of alternative therapeutic options, supportiveagent corticosteroid therapy has been provided to reduce vasculitis, and dextran 40 has been provided to decrease vascular thrombosis [2, 14]. Other therapeutic modalities are a plexus blockade resulting in vasodilatation (described in the literature after intra-arterial injection into the arteries of the upper extremity) and hyperbaric oxygen therapy, with good results used for ischemia of the hand [6, 7].

Although some case reports describe successful thrombolysis, many analyses showed bad outcome, and the affected extremities may require amputation of a finger, toe, hand, or foot—or even a major amputation $[1,11,12]$. Pratikto et al. [13] found that 15 of 40 addicted patients with an accidental intra-arterial injection of dissolved flunitrazepam in the upper extremities needed digit amputations, and two patients required major limb amputations. Nott et al. [6] mentioned that the outcome for the radial injection is worse compared with femoral artery injection. Treatment should begin as soon as possible after the arterial injection to prevent rhabdomyolysis and necrosis, and subsequent amputation.

In our case, the early intervention (4/h after the accidental intra-arterial injection) with a combination therapy of anticoagulation, vasodilatation, and local selective and superselective thrombolysis with urokinase led to complete resolution of the symptoms, and the limb was salvaged. Although intravenous heparinization has been broadly recommended, in our case, we performed intra-arterial heparinization via the sheath to inhibit further expansion of possible thrombus, which worked well. Because early arterial reocclusion after thrombolysis has been reported [5], we continued the therapy until we achieved a perfect result after 4 days, with permanent intensive care monitoring of the patient.

In conclusion, accidental intra-arterial injection of dissolved flunitrazepam tablets is a rare complication in intravenous drug addicts. The combination of anticoagulation, vasodilatation, and selective and superselective thrombolysis with urokinase is a valuable therapeutic strategy, and we were able to achieve complete cessation of the symptoms and salvage the leg.

Conflict of interest The authors declare that they have no conflict of interest.

\section{References}

1. Leifert JA, Bossaller L, Uhl M (2008) Acute ischaemia of the leg following accidental intra-arterial injection of dissolved flunitrazepam tablets. Vasa 37:374-378

2. Schulz S, Aksu T, Igde A et al (2002) Acute ischemia of the leg in a drug addict. Vasa 31:57-61

3. Dodd TJ, Scott RN, Woodburn KR et al (1994) Limb ischaemia after intra-arterial injection of temazepam gel: histology of nine cases. J Clin Pathol 47:512-514

4. Blair SD, Holcombe C, Coombes EN et al (1991) Leg ischaemia secondary to non-medical injection of temazepam. Lancet 338:1393-1394

5. Altstaedt HO (1999) Experience with the therapy of acute finger ischemia by high-dose intra-arterial infusion of PGE1 through a surgically introduced catheter. Chirurg 70:1144-1148

6. Nott DM, Chandrasekar R, Enabi L et al (1993) Intra-arterial injection of temazepam in drug abusers. Eur J Vasc Surg 7:87-89

7. Adir Y, Halpern P, Nachum Z et al (1991) Hyperbaric oxygen therapy for ischaemia of the hand due to intra-arterial injection of methadone and flunitrazepam. Eur J Vasc Surg 5:677-679

8. Andreev A, Kavrakov T, Petkov D et al (1995) Severe acute hand ischemia following an accidental intraarterial drug injection, successfully treated with thrombolysis and intraarterial Iloprost infusion. Case report. Angiology 46:963-967

9. Bittner C, Zuber M, Eisner L (2002) Acute ischemia of the hand in a drug addict after accidental intra-arterial injection. Swiss Surg 8:281-284

10. Goldberg I, Bahar A, Yosipovitch Z (1984) Gangrene of the upper extremity following intra-arterial injection of drugs. A case report and review of the literature. Clin Orthop Relat Res 188:223-229

11. Hering J, Angelkort B (2006) Acute ischemia of the hand after intra-arterial injection of flunitrazepam. Local combined fibrinolysis therapy in three cases. Dtsch Med Wochenschr 131:1377-1380

12. Joist A, Tibesku CO, Neuber M et al (1999) Gangrene of the fingers caused by accidental intra-arterial injection of diazepam. Dtsch Med Wochenschr 124:755-758

13. Pratikto TH, Strubel G, Biro F et al (2004) Intra-arterial injection of dissolved flunitrazepam tablets. Vasa 33:52-54

14. Treiman GS, Yellin AE, Weaver FA et al (1990) An effective treatment protocol for intraarterial drug injection. J Vasc Surg $12: 456-465$

15. Berger JL, Nimier M, Desmonts JM (1988) Continuous axillary plexus block in the treatment of accidental intraarterial injection of cocaine. N Engl J Med 318:930

16. Sommer CM, Radeleff BA (2009) A novel approach for percutaneous treatment of massive nonocclusive mesenteric ischemia: tolazoline and glycerol trinitrate as effective local vasodilators. Catheter Cardiovasc Interv 73:152-155

17. Tait IS, Holdsworth RJ, Belch JJ et al (1994) Management of intra-arterial injection injury with iloprost. Lancet 343:419 